\title{
Cutaneous Leishmaniasis of the Eyelids: A Case Series with Molecular Identification and Literature Review
}

\author{
Iraj Mohammadpour', Mohammad Hossein Motazedian,*, Farhad Handjani, Gholam Reza Hatam¹ \\ ${ }^{1}$ Department of Medical Parasitology and Mycology, ${ }^{2}$ Basic Sciences in Infectious Diseases Research Center, ${ }^{3}$ Molecular Dermatology Research \\ Center, Department of Dermatology, School of Medicine, Shiraz University of Medical Sciences, Shiraz, Iran
}

\begin{abstract}
Cutaneous leishmaniasis $(\mathrm{CL})$ is a protozoan disease which is endemic in Iran. It is transmitted by the Phlebotomus sand fly. The eyelid is rarely involved possibly because the movement of the lids impedes the sand fly from biting the skin in this region. Here, we report 6 rare cases of eyelid CL. The patients were diagnosed by skin scraping, culture, and PCR from the lesions. Skin scraping examination showed Leishmania spp. amastigotes in the cytoplasm of macrophages. Culture examination was positive for Leishmania spp. PCR was positive for Leishmania major and Leishmania tropica. The lesions were disguised as basal cell carcinoma, chalazion, hordeolum, and impetigo. The patients were treated with intramuscular meglumine antimoniate ( $20 \mathrm{mg} / \mathrm{kg} /$ day) for at least 3 weeks. They showed a dramatic response, and the lesions almost completely disappeared. We emphasized the importance of clinical and diagnostic features of lesions, characterized the phylogenetic relationship of isolated parasites, and reviewed the literature on ocular leishmaniasis.
\end{abstract}

Key words: Leishmania major, Leishmania tropica, cutaneous leishmaniasis, eyelid, PCR, Iran

\section{INTRODUCTION}

Leishmaniasis is a vector-borne parasitic disease caused by protozoans belonging to the genus Leishmania (Kinetoplastida: Trypanosomatidae). The disease is transmitted by infected sand flies (Diptera: Psychodidae) of the genus Phlebotomus in Old World and Lutzomyia in the New World [1]. Depending on the species of Leishmania involved, humans and a wide range of mammals can act as reservoirs [1]. There are 4 major clinical forms of this infection; cutaneous leishmaniasis (CL), either diffuse or localized, mucosal leishmaniasis (ML), visceral leishmaniasis (VL), fatal if untreated, and the post-kala azar dermal leishmaniasis (PKDL) [2]. In Iran, CL portrays a serious health problem, and is endemic in the central and southern parts of the country [3].

From the clinical perspective, CL is characterized by the progression of single or multiple localized lesions on exposed areas of skin that specifically ulcerate after an incubation period of 3 to 8 weeks. Inferior limbs, superior limbs, and the face are

\footnotetext{
• Received 22 July 2016, revised 5 September 2016, accepted 16 September 2016. *Corresponding author (motazedm@sums.ac.ir) (c) 2016, Korean Society for Parasitology and Tropical Medicine This is an Open Access article distributed under the terms of the Creative Commons Attribution Non-Commercial License (http://creativecommons.org/licenses/by-nc/4.0) which permits unrestricted non-commercial use, distribution, and reproduction in any medium, provided the original work is properly cited.
}

more commonly influenced, but ocular involvement is very rare. The eyelids, conjunctiva, and inner parts of the eye may be affected, and the lesions may imitate other more common lesions, such as chalazion, hordeolum, and impetigo [4-6]. The diagnosis of $\mathrm{CL}$ is made by the epidemiologic concept, clinical history and aspects of lesions, and demonstration of parasites by skin scraping, culture, or detection of parasite DNA [7]. Pentavalent antimonial compounds are the therapy of choice. The recommended dosage is $20 \mathrm{mg}$ per $\mathrm{kg}$ of body weight per day for at least 3 weeks [6-8].

In this report, we presented 6 rare cases of eyelid $\mathrm{CL}$, emphasized the importance of clinical and diagnostic features of lesions, characterized the phylogenetic relationship of isolated parasites, and reviewed the literature on ocular leishmaniasis.

\section{CASES PRESENTATION}

The studied patients were examined in the Dermatology Clinic of Saadi Hospital and Fajr Health Center from February to the end of December 2015. All patients proceeded from endemic regions of Fars province and patients had only eyelid lesions at presentation.

Written informed consents (code: IR.SUMS.REC.1394.S282) were obtained from the patients for publication of this case report and any accompanying images. Additional written in- 
formed consents were obtained from the parents of infant and child patients for publication of this case report and the accompanying images.

\section{Case 1}

An 80-year-old healthy woman stated a red nodule over the left upper eyelid that had slowly increased in size and ulcerated during the 4 months before her referral (Fig. 1A). During that period, she was treated with antibacterial and steroid ointments without improvement. The patient was suspected to have basal cell carcinoma and was referred for diagnosis and treatment. Clinical examination revealed an asymptomatic ul-
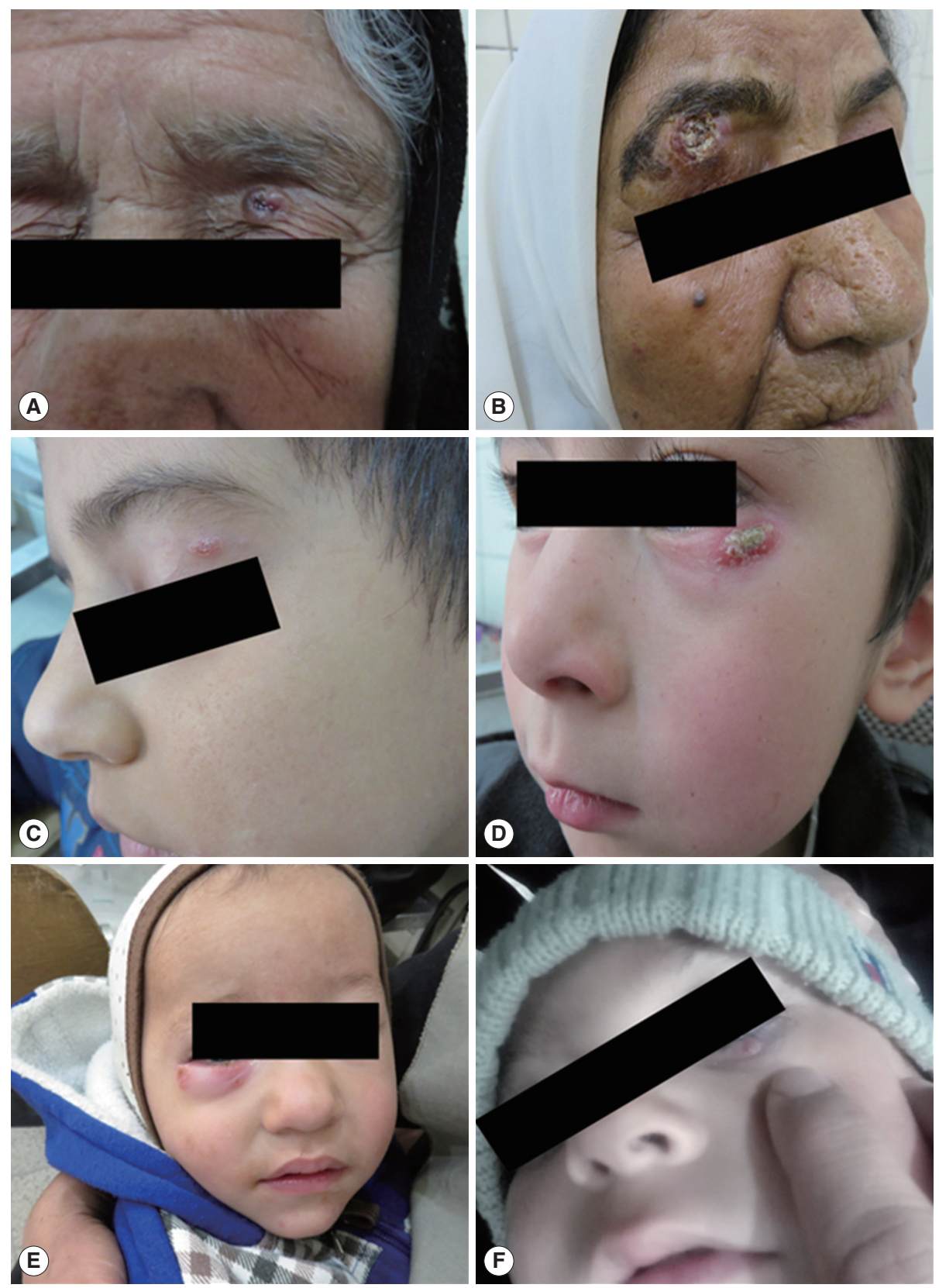

Fig. 1. Lesions of eyelid cutaneous leishmaniasis in our patients. (A) Left upper lid ulcerative nodule masquerading as basal cell carcinoma in case 1. (B) An erythematous right upper eyelid ulcerative plaque with overlying yellow crusting lesions in case 2. (C) An erythematous left superior eyelid ulcerated plaque lesion with skin flakes and mild ptosis in case 3. (D) An erythematous ulcerative plaque of the left lower eyelid concealed with yellow crusting lesions in case 4. (E) An erythematous right lower eyelid swelling with blepharoconjunctivitis in case 5. (F) A hordeolum-like nodule involving the left lower eyelid in case 6. 
cerative plaque with raised and erythematous borders. The visual acuity was not affected. Dermal scraping, culture, and PCR were performed. Touch impression smears and culture were negative for Leishmania species. The parasite was characterized by PCR as Leishmania major.

\section{Case 2}

A 74-year-old woman noted a small pimple on her right upper eyelid 2 months before presentation. The eyelid lesion enlarged slowly. Clinical examination revealed a well-defined, erythematous right upper eyelid ulcerative plaque with overlying yellow crusting lesions and mild to moderate ptosis (Fig. 1B). The remaining ophthalmologic and systemic studies were normal. Various treatments with antibiotics and steroids failed to heal the lesion. With a suspected diagnosis of a basal cell carcinoma, dermal scraping, culture, and PCR were achieved. Giemsa stain of the sample showed numerous leishman-bodies within the macrophages (Fig. 2), and the diagnosis of CL was confirmed. Culture was positive, as well, and the parasite was identified by PCR as L. major.

\section{Case 3}

A 7-year-old boy presented with a 6-week history of left upper eyelid lesion that initially was a small inflammatory papule and later became enlarged and ulcerated. Clinical examination showed an erythematous left superior eyelid ulcerated plaque lesion with skin flakes and mild ptosis (Fig. 1C). There was no corneal involvement, and the ocular examination was normal. Giemsa staining revealed macrophage-filled amasti-

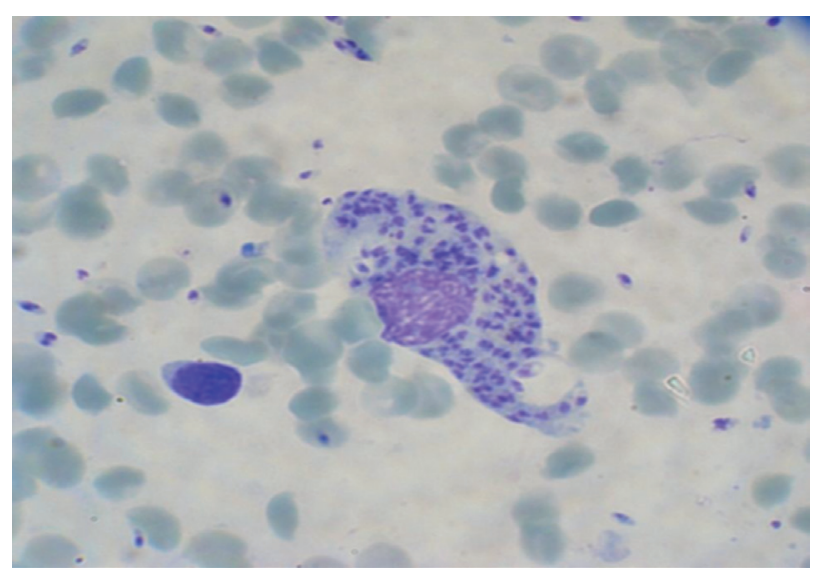

Fig. 2. Touch impression smear in case 2. Numerous intracellular and scattered extracellular amastigotes are seen (Giemsa stain; original magnification, $\times 1,000)$. Kinetoplasts are visible in many amastigotes. gotes. Culture was positive for Leishmania spp. Subsequent identification of culture by PCR classified the parasite as L. major.

\section{Case 4}

A 5-year-old boy demonstrated with a 1-year history of left lower eyelid erythema and induration resistant to treatment with antibiotic ointments. The lesions gradually progressed to ulcerative plaque within 3 months. Clinical examination revealed 1 erythematous ulcerative plaque covered with yellow crusting lesions (Fig. 1D). There was no corneal involvement, and the ocular examination was normal. Touch impression smears and culture were negative. PCR characterized the parasite as L. major.

\section{Case 5}

A healthy 14-month-old boy presented with a 6-week history of ruddiness and protuberance on the right lower eyelid that was initially diagnosed as impetigo, chalazion, or hordeolum. He received multiple courses of topical antibacterial therapy. External examination revealed erythematous right lower eyelid swelling with blepharoconjunctivitis (Fig. 1E). The remainder of his comprehensive eye examination was normal. In dermal scraping smear stained with Giemsa stain, amastigotes were seen along with some neutrophils and macrophages. Culture was positive, as well. PCR characterized the parasite as Leishmania tropica.

\section{Case 6}

A 7-month-old girl pursued treatment 4 weeks after the appearance of a lesion that primarily was a small papule at the left inferior eyelid and later became nodular. Initially, she had been assessed by an ophthalmologist, was diagnosed as hordeolum and was treated with antibiotic eye drops and ointments, but no improvement was noticed. Dermatologic examination disclosed 1 hordeolum-like nodule involving the left lower eyelid (Fig. 1F). Slit dermal smears and culture were positive. PCR depicted the parasite as L. tropica.

\section{MATERIALS AND METHODS}

For the margin dermal scraping, a no. 15 disposable sterile surgical blade (Unicut, Chicago, Illinois, USA) was used to make a slit in the border of the lesion. Dermal tissue from the wall of the slit was scraped with the blade and smeared on 2 glass slides. A total of 3 scrapings from the slit were smeared 
on a single slide. The touch preparation of skin scrapings were stained with Giemsa (Merck, Darmstadt, Germany), and finally examined for amastigotes by microscopy. Using Giemsa, amastigotes are seen within the cytoplasm of macrophages as pale blue oval bodies with a dark blue nucleus and a small rod-shaped kinetoplast with a specialized mitochondrial structure that contains extra-nuclear DNA.

In addition, an aspirate from the active indurated margins of the same lesions was transferred to 2 tubes of the biphasic modified NNN culture medium. The cultures were kept at 22$25^{\circ} \mathrm{C}$ and sub-cultured in RPMI-1640 medium (Gibco, Frankfurt, Germany) supplemented with 15\% heat inactivated fetal calf serum (Gibco), $100 \mathrm{U} / \mathrm{ml}$ penicillin, and $100 \mu \mathrm{g} / \mathrm{ml}$ streptomycin (Gibco) for mass cultivation.

Total genomic DNA was extracted from each clinical sample using the AccuPrep ${ }^{\circledR}$ Genomic DNA Extraction Kit (Bioneer, Daejeon, Korea), according to the manufacturer's instructions. The quantification and quality control of the DNA extraction procedures were performed using a nano spectrophotometer (NanoDrop 1000, Thermo Fisher Scientific, Waltham, Massachusetts, USA). The DNA was stored at $-20^{\circ} \mathrm{C}$ until being used.

The conserved area of the minicircle kDNA from the Leishmania species was amplified by semi-nested PCR. The primers LINR4 (forward) (5'-GGG GTT GGT GTA AAA TAG GG-3'), LIN17 (reverse) (5'-TTT GAA CGG GAT TTC TG-3'), and LIN19 (reverse) (5'-CAG AAC GCC CCT ACC CG-3') were used for amplification [9]. The reaction mixtures were incubated at $94^{\circ} \mathrm{C}$ for $5 \mathrm{~min}$, followed by 40 cycles, each consisting of $30 \mathrm{sec}$ at $94^{\circ} \mathrm{C}, 30 \mathrm{sec}$ at $52^{\circ} \mathrm{C}$ (for LINR4 and LIN17) or $58^{\circ} \mathrm{C}$ (for LINR4 and LIN19), and $1 \mathrm{~min}$ at $72^{\circ} \mathrm{C}$, and a final extension at $72^{\circ} \mathrm{C}$ for $10 \mathrm{~min}$. PCR products were visualized by UV after electrophoresis on 1.5\% agarose gel using TAE buffer and staining with GelRed (Biotium Inc., Hayward, California, USA). A 650-bp fragment was amplified for L. major, whereas a 760-bp fragment was amplified for L. tropica (Fig. 3). All primers were synthesized by Macrogen Genomics Laboratories (Macrogen, Seoul, Korea).

For confirmation, the PCR products were purified by using QIAquick Gel Extraction Kit (QIAGEN, Hilden, Germany) and sequenced on both ends through the sequencing service of Macrogen Genomics Laboratories (Macrogen). The resulting sequences were edited and aligned with the BioEdit, version 7.2.5 [10]. Multiple alignments were performed with data related to Leishmania species from Iran and other countries deposited in GenBank.

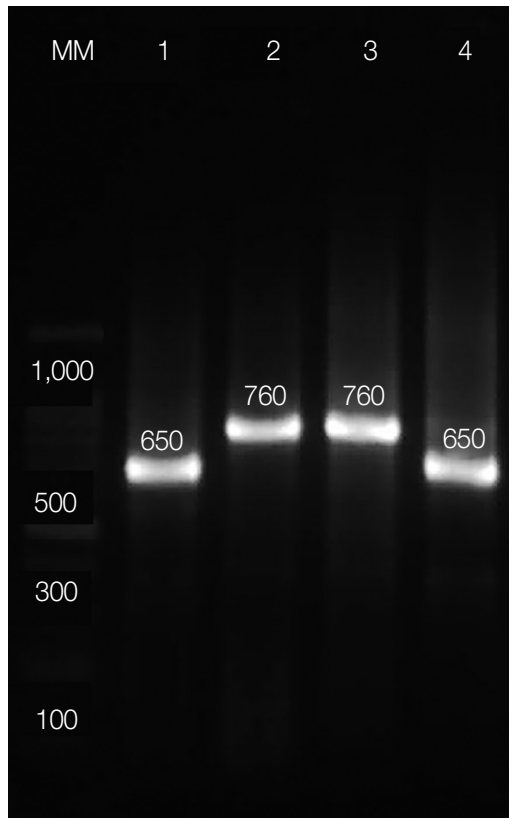

Fig. 3. Electrophoresis of PCR products of DNA extracted from positive smears and cultures. The 4 lanes contained the products from positive controls of L. tropica (lane 3) and L. major (lane 4), ocular lesions due to $L$. major and $L$. tropica (lanes 1 and 2, respectively), and a molecular size ladder (MM).

A phylogenetic tree was constructed via maximum composite likelihood option of the neighbor-joining (NI) method using MEGA-7 [11]. The sequences of 4 patients of L. major showed $100 \%$ identity to the published isolate IranJWmaj (accession no. AB678349). In addition, the 4 L. major showed $99 \%, 99 \%$, and $98 \%$ identity to the published isolates from UK and Iran (nos. AF308685, KM555292, and KM555295), respectively. The sequences of 2 patients of $L$. tropica showed $100 \%$ identity to the published isolate IranJWtrop (no. AB678350). Also, these 2 isolates showed 99\% identity to the published isolates from UK, Egypt, and Iran (nos. AF308689, X84845, and KM491168). NJ method grouped the 6 patients into 2 clusters. Cluster I contained isolated L. major from the 4 eyelid CL patients in which these 4 isolates belonged to the same geographical origin. Cluster II contained isolated $L$. tropica from the 2 eyelid CL patients in which both isolates also belonged to the same geographical area (Fig. 4).

In a final manner, infants were treated with $20 \%$ paromomycin ointment with complete healing after 2 months. The remaining patients were treated systemically with meglumine antimoniate (Glucantime ${ }^{\circledR}$ ), $20 \mathrm{mg} / \mathrm{kg} /$ day over a 3-week period with good results $[6,8]$. All lesions resolved after 3 weeks of therapy. After 1 year of follow up, the patients were completely 


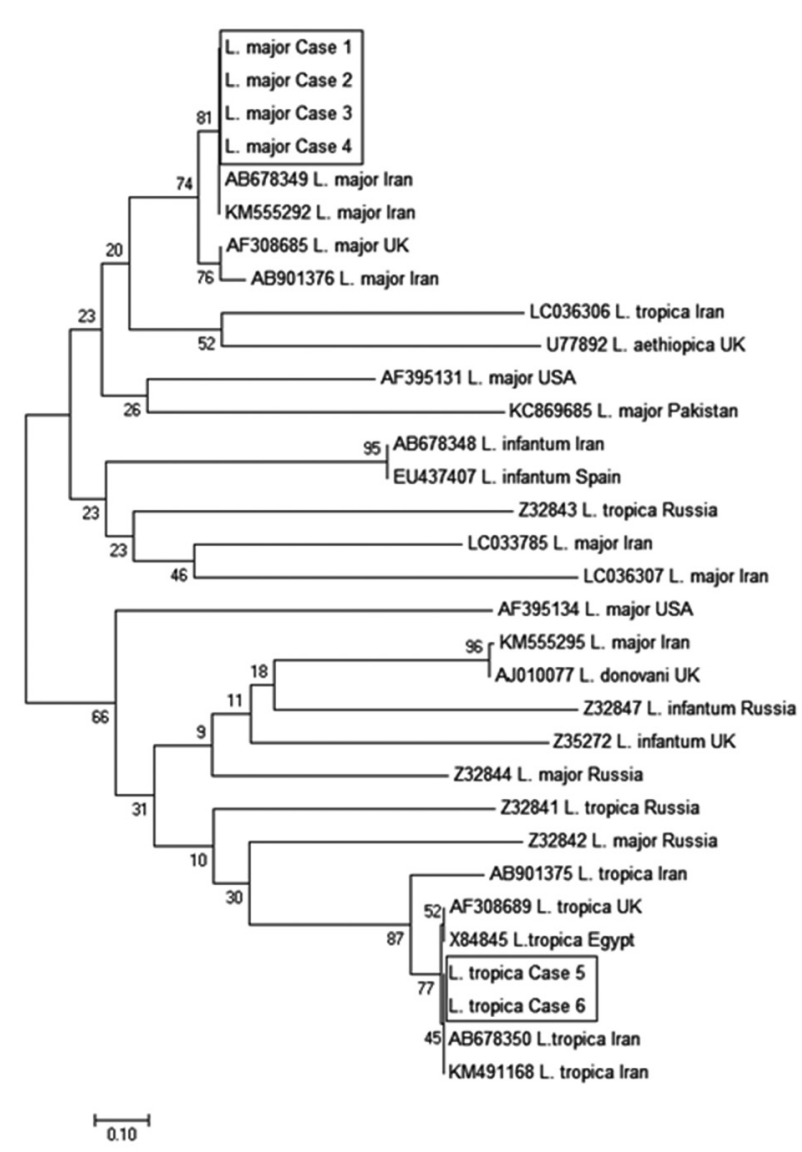

Fig. 4. Phylogenetic relationship among various Leishmania species to each other as inferred by neighbor-joining tree based on minicircle kDNA gene. Numbers on branches are percentage bootstrap values of 1,000 replicates. All positions with less than 95\% site coverage were eliminated. The evolutionary distances between sequences were computed using the maximum composite likelihood method and are in the units of the number of base substitutions per site. The scale bar indicates an evolutionary distance of 0.10 nucleotides per position in the sequence. The reference sequences accession numbers are inserted. Evolutionary analyses were conducted in MEGA-7.

normal, and no relapses were observed.

\section{DISCUSSION}

Leishmaniasis is a group of diseases caused by a protozoan parasite of the genus Leishmania and transmitted by sand flies. CL is caused by different species of Leishmania with different clinical features [1]. CL is endemic in Iran and usually caused by $L$. major or $L$. tropica. Clinical forms of localized CL are papular, nodular, ulcerative plaque, infiltrative, and impetiginoid. In immunocompromised patients, fulminant cutaneous and ocular leishmaniasis may develop [12].
Ocular involvement is rare. It is appraised that in CL the eyelid is involved in $0.4 \%$ of cases. The low occurrence on the eyelid is ascribed to the regular movements of the eyelid, which hinder the sand fly from biting the skin in this region [5]. It initially exhibits as highly polymorphous lesions, such as swelling [13], papules, and plaques [14]. A few cases of eyelids $\mathrm{CL}$, which presented nodules covered by crusts and scaling, have been reported $[15,16]$. They can imitate other conditions, such as chalazion $[4,5]$, hordeolum $[5,6]$, infundibular cyst [17], and tumors which masquerading as basal cell carcinoma or keratoacanthoma in elderly patients $[15,16,18]$.

Ocular manifestations of leishmaniasis that have been reported includes uni- or bilateral ptosis, lagophthalmia, blepharoconjunctivitis [19,20], ulcerative blepharitis [13], conjunctivitis [5,6], nodular episcleritis [6], scleromalacia [21], intraretinal hemorrhage [22], and optic neuropathy [23]. In endemic areas, such manifestations as the ulcerated lesion of the eyelid in our patient should raise the suspicion of leishmaniasis and be included in the differential diagnosis. Ocular complications comprises as palpebral and conjunctival scarring [5], trichiasis [5], eyelash loss [5], eyelid ectropion [15], dacryocystitis [14], scleral perforation [21], corneal opacity [5], and the development of squamous cell carcinoma [24].

In endemic regions and in travelers from endemic territories, it is essential for the physician to be attentive that any unusual lesion should be examined carefully for cutaneous leishmaniasis [25]. Our patients had been erroneously treated for bacterial infection, and eventually the diagnoses were based on the presence of amastigotes in lesion, and positive outcome of culture or PCR.

Despite the fact that the leishmanial infection is self-limited in most immunocompetent patients, medicinal treatment of the disease engages systemic or local use of pentavalent antimonials and aromatic diamidines. Lesions on cosmetically or functionally important sites (such as the face or hands), those with associated lymphangitis, and multiple or persistent lesions are best given active treatment [16]. Early detection of the infection is essential in order to begin impressive treatment and deter more serious complications. If eyelid lesions abide untreated, adjacent spread may extend to the conjunctiva, episclera, and cornea, with progression of interstitial keratitis [6]. Residual scarring and deformity may necessitate surgical amendment [5]. Hence, ocular leishmaniasis is regarded potentially a blinding disorder. Ocular leishmaniasis can be treated by $20 \%$ paromomycin ointment, and pentavalent anti- 
monials (intralesional and/or intramuscular Pentostam ${ }^{\circledR}$ or Glucantime ${ }^{\circledR}$ ). The recommended dosage is $20 \mathrm{mg} / \mathrm{kg} /$ day over 3 weeks [8].

In summary, we present 6 uncommon cases of eyelid leishmaniasis masquerading as basal cell carcinoma, chalazion, hordeolum, and impetigo. Physicians should embrace CL in the differential diagnosis of patients suffering from chronic granulomatous lid lesions in regions where this disease is endemic.

\section{ACKNOWLEDGMENTS}

The results described in this paper were a part of Ph.D. thesis of Iraj Mohammadpour. The study was financially supported by the office of vice-chancellor for research of Shiraz University of Medical Sciences, Iran (grant no. 94-7548).

\section{CONFLICT OF INTEREST}

The authors declare that there is no conflict of interest regarding the publication of this paper.

\section{REFERENCES}

1. Murray HW, Berman JD, Davies CR, Saravia NG. Advances in leishmaniasis. Lancet 2005; 366: 1561-1577.

2. Alvar J, Vélez ID, Bern C, Herrero M, Desjeux P, Cano J, Jannin J, den Boer M. Leishmaniasis worldwide and global estimates of its incidence. PLoS ONE 2012; 7: e35671.

3. Davami MH, Motazedian MH, Kalantari M, Asgari Q, Badzohre A, Mohammadpour I. First microscopical and molecular-based characterization of Leishmania major within naturally infected Phlebotomus salehi (Diptera; Psychodidae) in Fars province, southern Iran. Ann Trop Med Parasitol 2011; 105: 485-491.

4. Rahimi M, Moinfar N, Ashrafi A. Eyelid leishmaniasis masquerading as chalazia. Eye (Lond) 2009; 23: 737.

5. Sadeghian G, Nilfroushzadeh MA, Moradi SH, Hanjani SH. Ocular leishmaniasis: a case report. Dermatol Online J 2005; 11: 19 .

6. Abrishami M, Soheilian M, Farahi A, Dowlati Y. Successful treatment of ocular leishmaniasis. Eur J Dermatol 2002; 12: 88-89.

7. Masmoudi A, Hariz W, Marrekchi S, Amouri M, Turki H. Old World cutaneous leishmaniasis: diagnosis and treatment. J Dermatol Case Rep 2013; 7: 31-41.

8. Monge-Maillo B, López-Vélez R. Therapeutic options for Old World cutaneous leishmaniasis and New World cutaneous and mucocutaneous leishmaniasis. Drugs 2013; 73: 1889-1920.

9. Aransay AM, Scoulica E, Tselentis Y. Detection and identification of Leishmania DNA within naturally infected sand flies by seminested PCR on minicircle kinetoplastic DNA. Appl Environ Microbiol 2000; 66: 1933-1938.

10. Hall TA. BioEdit: a user-friendly biological sequence alignment editor and analysis program for Windows 95/98/NT. Nucleic Acids Symp Ser 1999; 41: 95-98.

11. Kumar S, Stecher G, Tamura K. MEGA7: Molecular Evolutionary Genetics Analysis version 7.0 for bigger datasets. Mol Biol Evol 2016; 33: 1870-1874.

12. Meenken C, van Agtmael MA, Ten Kate RW, van den Horn GJ. Fulminant ocular leishmaniasis in an HIV-1-positive patient. AIDS 2004; 18: 1485-1486.

13. Jafari AK, Akhyani M, Valikhani M, Ghodsi ZS, Barikbin B, Toosi S. Bilateral cutaneous leishmaniasis of upper eyelids: a case report. Dermatol Online J 2006; 12: 20.

14. Durdu M, Gökçe S, Bagirova M, Yalaz M, Allahverdiyev AM, Uzun S. Periocular involvement in cutaneous leishmaniasis. J Eur Acad Dermatol Venereol 2007; 21: 214-218.

15. Oliveira-Neto MP, Martins VJ, Mattos MS, Pirmez C, Brahin LR, Benchimol E. South American cutaneous leishmaniasis of the eyelids: report of five cases in Rio de Janeiro State, Brazil. Ophthalmology 2000; 107: 169-172.

16. Mencía-Gutiérrez E, Gutiérrez-Díaz E, Rodríguez-Peralto JL, Monsalve-Córdova J. Old World eyelid cutaneous leishmaniasis: a case report. Dermatol Online J 2005; 11: 29.

17. Veraldi S, Bottini S, Currò N, Gianotti R. Leishmaniasis of the eyelid mimicking an infundibular cyst and review of the literature on ocular leishmaniasis. Int J Infect Dis 2010; 14 (suppl): 230-232.

18. Jaouni T, Deckel Y, Frenkel S, Ilsar M, Pe'er J. Cutaneous leishmaniasis of the eyelid masquerading as basal cell carcinoma. Can J Ophthalmol 2009; 44: 47-48.

19. Satici A, Gurler B, Aslan G, Ozturk I. Ocular involvement in cutaneous leishmaniasis: four cases with blepharoconjunctivitis. Eur J Epidemiol 2004; 19: 263-266.

20. Ayele FA, Wolde YA, Hagos T, Diro E. Ocular leishmaniasis presenting as chronic ulcerative blepharoconjunctivitis: a case report. J Clin Exp Ophthalmol 2015; 6: e1000395.

21. Reinecke P, Gabbert HE, Strunk W, Lösche CC. Ocular scleromalacia caused by leishmaniasis: a rare cause of scleral perforation. Br J Ophthalmol 2001; 85: 240-241.

22. Montero JA, Ruiz-Moreno JM, Sanchis E. Intraretinal hemorrhage associated with leishmaniasis. Ophthalmic Surg Lasers Imaging 2003; 34: 212-214.

23 Huna-Baron R, Warren FA, Miller W, Jacobs J, Green J, Kupersmith MJ. Mucosal leishmaniasis presenting as sinusitis and optic neuropathy. Arch Ophthalmol 2000; 118: 852-854.

24. Matayoshi S, Baddini-Caramelli C, Goldbaum M, Takei LM, Honda M, Kara-José N. Epidermoid carcinoma arising in an ocular Leishmania lesion. Br J Ophthalmol 2000; 84: 1331-1332.

25. Vinetz JM, Soong L. Leishmania mexicana infection of the eyelid in a traveler to Belize. Braz J Infect Dis 2007; 11: 149-152. 